\title{
Die ersten 24 Stunden - der akute Thoraxschmerz
}

\author{
The first 24 hours - acute chest pain
}

Autoren

Institut

\section{S. Baumanns ${ }^{1}$ J. vom Dahl}

${ }^{1}$ Klinik für Kardiologie - Innere Medizin II, Kliniken Mariahilf GmbH, Mönchengladbach
Intensivmedizin,

Notfallmedizin

\section{Schlüsselwörter}

akuter Thoraxschmerz

akutes Koronarsyndrom

akutes Aortensyndrom

Lungenembolie

Pneumothorax

Brustschmerzambulanz

$\checkmark$ kardiogener Schock

\section{Keywords}

acute chest pain

acute coronary syndrome

acute aortic syndrome

pulmonary embolism

Dneumothorax

chest pain unit

- cardiogenic shock

eingereicht 23.11.2011

akzeptiert 14.06.2012

\section{Bibliografie}

DOI 10.1055/s-0032-1305089

Dtsch Med Wochenschr 2012;

137: 1468-1473 - (c) Georg

Thieme Verlag KG · Stuttgart .

New York · ISSN 0012-0472

Korrespondenz

Dr. med. S. Baumanns

Klinik für Kardiologie Innere Medizin II,

Kliniken Mariahilf GmbH

Viersener Str. 450

41063 Mönchengladbach

eMail Stefan.Baumanns@

mariahilf.de

\section{Überblick}

\section{$\nabla$}

Thorakale Schmerzen sind nach abdominellen Schmerzen der zweithäufigste Grund für den Arztkontakt im Krankenhaus und stellen den Behandler aufgrund der Vielzahl an Differenzialdiagnosen vor eine große Herausforderung. Die Spannweite der möglichen Grunderkrankungen reicht von der harmlosen Interkostalneuralgie über die Ösophagusruptur bis zum akuten ST-Hebungsinfarkt und akuten Aortensyndrom. Die Aufgabe des erstbehandelnden Arztes ist neben der schnellen Diagnose vor allem eine rasche Risikostratifizierung des Patienten und Durchführung bzw. Veranlassung der notwendigen therapeutischen Maßnahmen.

\section{Ursachen}

\section{$\nabla$}

Knapp ein Viertel aller Patienten mit Thoraxschmerzen (24\%) haben ein akutes Koronarsyndrom, weitere $17 \%$ neuroradikuläre Beschwerden, $10 \%$ Lungenerkrankungen. Je weitere $6 \%$ machen Herzrhythmusstörungen und Herzinsuffizienz-Symptome aus. Hypertensive Krise, Lungenembolie und Pneumothorax liegen mit 1-2\% ebenso wie die Pleuritis im unteren Bereich der Auftretenshäufigkeit [10]. Akute Aortensyndrome (v.a. eine thorakale Aortendissektion) zählen mit ca. $0,6 \%$ zu den seltenen Ursachen (1) Tab.1).

Seit Einführung der (standardisierten) Zertifizierung von Chest Pain Units (CPU) durch die Deutsche Gesellschaft für Kardiologie (139 CPU, Stand 26.3.2012 [Quelle: cpu.dgk.org]) hat sich die Versorgung dieser Patienten durch die Entwicklung von strukturierten diagnostischen und therapeutischen Algorithmen und standardisierten apparativen Ausstattungsbedingungen dieser Spezial-Ambulanzen deutlich verbessert. Zudem profitieren die Kliniken in Zeiten pauschalisier- ter Vergütungssysteme von einer effizienteren Patientenversorgung durch Verkürzung der Liegezeiten.

Aus strategischen und praktischen Erwägungen muss bei der differenzialdiagnostischen Abklärung zunächst der Fokus auf potenziell vital gefährdende Grunderkrankungen gelegt werden.

\section{Potenziell lebensbedrohlich} $\nabla$

\section{Akutes Koronarsyndrom}

Zum akuten Koronarsyndrom (ACS) gehören der ST-Hebungsinfarkt (STEMI) und der Nicht-STHebungsinfarkt (NSTEMI) - beide gekennzeichnet durch die Freisetzung kardialer Biomarker (i.e.S.Troponin I/T) - sowie die instabile Angina pectoris und die Postinfarktangina. Die jährliche Inzidenz liegt bei ca. 3 Patienten pro 1000 Einwohner [23]. Ca. 1-4\% aller Patienten, die sich wegen eines STEMI/NSTEMI in der Notaufnahme einer Klinik vorstellen, werden dort nicht diagnostiziert; die Anzahl steigt, rechnet man Patienten mit instabiler Angina pectoris hinzu. Ca. 8\% der Patienten mit einem akuten Koronarsyndrom werden fälschlicherweise aus deutschen Notaufnahmen entlassen und haben eine 30Tage-Mortalität von 11-25\% [18].

Klinisches Leitsymptom ist der akute Brustschmerz mit einer Dauer von mehr als 20 Minuten, in der Regel mit Ausstrahlung in den linken Arm, Nacken oder Hals. Vegetative Begleitsympto-

Tab.1 Differenzialdiagnose thorakaler Schmerzen [9].

$\begin{array}{ll}\text { kardiale Ursachen } & \text { nicht kardiale Ursachen } \\ \text { ischämisch } & \text { gastroösophageal } \\ \text { nicht-ischämisch } & \text { pulmonal } \\ & \text { muskuloskelettal } \\ & \text { neural }\end{array}$


me (Schweißausbruch, Übelkeit, Erbrechen) resultieren aus dem Schmerz sowie der vegetativen Dysregulation und kommen in unterschiedlicher Häufigkeit vor. Dyspnoe sowie Schmerzausstrahlung ins Epigastrium sind typisch bei jüngeren Patienten (25-40 Jahre), älteren Frauen ( $>75$ Jahre) und bei Patienten mit Diabetes oder chronischer Niereninsuffizienz [6].

\section{Akutes Aortensyndrom}

Das akute Aortensyndrom (im engeren Sinne: die thorakale Aortendissektion) ist mit ca. 0,6\% aller Patienten mit akutem Thoraxschmerz eine seltene Ursache. Da die klinischen Kriterien für dieses Krankheitsbild aufgrund der vielen unterschiedlichenSymptome nur schwer definierbar sind, ist eine rasche Diagnosestellung schwer: Nach Kodolitsch wird die Verdachtsdiagnose „akute Aortendissektion“ in 99\% der vermuteten Fälle nicht bestätigt, andererseits werden bis zu 35\% aller Patienten nicht oder erst verspätet korrekt diagnostiziert [10]. Mit einer unbehandelten Letalität von ca. $1 \%$ pro Stunde ist insbesondere eine Mitbeteiligung der Aorta ascendens in die Dissektion eine vital bedrohliche Erkrankung.

Leitsymptom (90\%) ist der akut einsetzende vernichtende Thoraxschmerz, der reißenden Charakter besitzt und oftmals zwischen die Schulterblätter ausstrahlt, oft gefolgt von einem beschwerdefreien Intervall [20]. Ca. 30\% der Patienten berichten über Ischämiesymptome der unteren Extremitäten. Neurologische Defizite werden in bis zu 17\% der Betroffenen beobachtet und deuten auf eine Beteiligung der hirnversorgenden Arterien hin [10]. In sehr seltenen Fällen können Schmerzen auch fehlen und neurologische Ausfälle das alleinige Symptom einer Aortendissektion darstellen. Zeichen einer akuten Aortenklappeninsuffizienz oder oberen Einfluss-Stauung infolge eines tamponierenden Perikardergusses gehen oftmals mit klinischen Zeichen des kardiogenen Schocks einher und sind prognostisch ungünstig.

\section{Lungenembolie}

Die jährliche Inzidenz von venösen Thromboembolien (VTE) liegt bei ca. 150-200 Fällen pro 100000 Einwohner in den westlichen Industrieländern [11]. Die mittlere Letalität beträgt ca. $11 \%$ innerhalb der ersten 14 Tage, mithin ca. 40000 Todesfälle allein in Deutschland.

Die Verdachtsdiagnose begründet sich auf einer Vielzahl unterschiedlicher Symptome: Neben akut einsetzender Dyspnoe (80\%) und pleuritischen/retrosternalen, atemabhängigen Schmerzen (52\%) können Husten (20\%), Synkope (19\%) und Hämoptysen (11\%) als Leitsymptom auftreten. Begleitend sind oft Tachypnoe (70\%) und Tachykardie $(26 \%)$ zu beobachten $[14,21]$

\section{Spontan-Pneumothorax}

Die Differenzierung in einen primären bzw. sekundären Spontan-Pneumothorax beruht auf der Abwesenheit bzw. Präsenz einer vorbestehenden Lungenerkrankung (meist COPD) [3]. Typische Symptome sind der akut einsetzende unilaterale stechende Thoraxschmerz, verbunden mit akuter Dyspnoe. Meist besteht darüber hinaus ein trockener Husten. Tachypnoe und Tachykardie können - je nach Ausprägung des Krankheitsbildes - unspezifische Begleitsymptome sein. Die Intensität der Beschwerden variiert stark, die unspezifischen Symptome machen eine Diagnose allein anhand klinischer Parameter schwierig.

\section{Ösophagus-Ruptur (Boerhaave-Syndrom)}

Die spontane Ösophagusruptur ist eine seltene Ursache des akuten Thoraxschmerzes. Sie betrifft in 90\% der Fälle die linkslaterale Wand des distalen Ösophagusdrittels [13] und hat unbehandelt eine Mortalität von 20-40\% [19]. Typische Symptome der schwer kranken Patienten sind ein akut einsetzender Thoraxschmerz, oft verbunden mit einer Schock-Konstellation in Verbindung mit akuter Dyspnoe und heftigem Erbrechen. Die Prognose ist abhängig vom Zeitraum zwischen Symptombeginn und Diagnosestellung/Therapie sowie von etwaigen Komorbiditäten.

\section{kurzgefasst}

Akute Koronarsyndrome liegen bei knapp einem Viertel aller Patienten mit Thoraxschmerz vor. Neben einer Differenzierung zwischen kardialen und nichtkardialen Ursachen ist eine rasche Risikostratifizierung prognostisch entscheidend.

\section{Potenziell nicht akut lebensbedrohlich}

\section{$\nabla$}

Akute Pankreatitis

Eine akute Pankreatitis ist aufgrund ihrer meist typischen klinischen Symptomatik - dumpfe gürtelförmige Oberbauchschmerzen mit plötzlichem Beginn und langsam steigernder Intensität, die nach Erreichen ihres Maximums meist anhalten, begleitet von Erbrechen und Fieber - in den wenigsten Fällen Grund zur Abklärung eines Thoraxschmerzes. Klinisch imponiert bei ca. $60 \%$ der Patienten das typische Bild des „Gummibauches“; radiologisch können häufig Zeichen eines paralytischen Subileus diagnostiziert werden. Aufgrund der selten auch in den Rücken ausstrahlenden Schmerzen werden die Patienten nicht selten aber auch unter dem Bild eines akuten Koronarsyndroms vorstellig.

Neben den klinischen und anamnestischen Angaben gibt es eine Vielzahl von Laborparametern, von denen zur Diagnosesicherung die Serum-Lipase die höchste Sensitivität und Spezifität (jeweils 80-100\%) aufweist [2]. Eine biliäre Genese ist die häufigste Ursache (ca. 60\%) und muss durch Bestimmung der Cholestase-Parameter sowie Oberbauch-Sonographie von anderen Formen abgegrenzt werden.

\section{Pneumonie/Pleuritis}

Die Häufigkeit ambulant erworbener Pneumonien (sogenannte Community acquired pneumonia, CAP) liegt bei ca. 400000 600000 Patienten pro Jahr. Es handelt sich dabei definitionsgemäß um eine akute mikrobielle Infektion des Lungenparenchyms bei Erwachsenen ohne Abwehrschwäche und unter Ausschluss einer im Krankenhaus bzw. innerhalb der ersten 4 Wochen nach Entlassung erworbenen Pneumonie [7].

Während die Letalität bei ambulant behandelten Patienten mit 1\% niedrig ist, steigt sie bei stationär behandelten/behandlungsbedürftigen Patienten auf $13-14 \%$ an. Ursache ist der unterschiedliche Schweregrad der CAP; durch Einführung klinischer ScoringSysteme (z.B. mod. ATS-Score, CRB-65-Score), die krankheitsassoziierte Komplikationsrate und die Pneumonie-assoziierte Sterblichkeit sowie individuelle Risikofaktoren erfassen, kann die Notwendigkeit einer Krankenhauseinweisung objektiviert werden. 
Das klinische Erscheinungsbild variiert in hohem Ausmaß: Neben Dyspnoe sowie produktivem oder unproduktivem Husten stellen thorakale Schmerzen ein häufiges Leitsymptom dar. Diese sind oft - v.a. bei Vorliegen einer begleitenden Pleuritis atemabhängig, oftmals aber auch - z.B. bei bilateralem Befall nicht exakt lokalisierbar. Je nach Schwere der Infektion kann der klinische Aspekt von einer leichten Störung des Allgemeinbefindens bis hin zum Vollbild des septischen Schocks und schwerer respiratorischer Insuffizienz reichen. Fieber liegt nur bei wenigen Patienten vor und ist diagnostisch allein nicht verwertbar.

Ösophagitis und gastroösophageale Refluxkrankheit Nach den Leitlinien der deutschen Gesellschaft für Verdauungsund Stoffwechselerkrankungen liegt eine gastroösophageale Refluxkrankheit (GERD) vor, wenn „ein Risiko für organische Komplikationen durch einen gesteigerten gastroösophagealen Reflux und/oder eine signifikante Störung des gesundheitsbezogenen Wohlbefindens (Lebensqualität) infolge der Refluxbeschwerden besteht“. Darunter werden verschiedene Manifestationen zusammengefasst: nicht-erosive sowie erosive Refluxkrankheit, Barrett-Ösophagus und extraösophageale Formen [12]. Wegen der hohen Prävalenz dieser Erkrankung in den westlichen Industrieländern mit oftmals unspezifischen Beschwerden muss die GERD immer als Differenzialdiagnose berücksichtigt werden.

\section{Costochondritis/Tietze-Syndrom, Radikulopathien}

Bei der Costochondritis liegt eine Entzündung des Knorpels der Costosternal- oder Costochondralgelenke der Rippen vor. Sie wird meist durch Systemerkrankungen (z.B. Morbus Reiter) verursacht und oft mit dem sogenannten Tietze-Syndrom verwechselt. Diesem liegt jedoch keine eigentliche Entzündungsreaktion zugrunde, vielmehr handelt es sich um synchondrosennahe Mikrofrakturen im Bereich der oberen Sternokostalgelenke, hervorgerufen meist durch Ermüdungs- oder Überlastungsreaktionen im Rahmen körperlicher Belastung oder auch einer Schwangerschaft. Nach Fruergaard sind muskuloskelettale Beschwerden bei bis zu 28\% der Patienten Grund für die Aufnahme in einer CPU [5].

\section{Herpes zoster}

Der Herpes zoster ist eine Virusinfektion, die mit Schmerzen, Empfindungsstörungen und typischen pustulösen Hautveränderungen einhergeht und typischerweise unilateral das Versorgungsgebiet eines oder mehrerer Hautnerven (sog. Dermatome) betrifft. Betroffen sind vor allem ältere und immungeschwächte Menschen, die Erkrankung betrifft aber auch Immunkompetente und jüngere Erwachsene. Am häufigsten betroffen sind die Dermatome T3-L3. Insbesondere bei Erwachsenen führt die akute Neuritis zu heftigen Schmerzen, die - sofern thorakale Dermatome betroffen sind - als Thoraxschmerz imponieren. Die Diagnose wird meist durch das typische klinische Erscheinungsbild gestellt, gelegentlich gehen die Schmerzen dem pathognomonischen Exanthem aber um kurze Zeit voraus.

\section{Psychogener Thoraxschmerz}

Psychogene Faktoren spielen in der Genese des Thoraxschmerzes eine wesentliche Rolle: Sie können einerseits parallel zu anderen Ursachen thorakaler Schmerzen auftreten und deren Wahrnehmung und die Intensität zum Teil erheblich beeinflussen. Andererseits begünstigen generalisierte Angst- und Panikstörungen das Auftreten eines funktionellen Thoraxschmerzes und stellen damit eine eigenständige Ursache dar [4]. Die Inzidenz schwankt in verschiedenen Studien sehr stark (ca. 7-17\%). Ca. 20\% dieser Patienten leiden unter Panikstörungen. Das Durchschnittsalter liegt bei 45 Jahren, die Prävalenz ist bei Frauen etwas höher als bei Männern. Die Beschreibung atypischer Angina-pectoris-Beschwerden kann einen Hinweis auf eine psychogenen Komponente geben, spezifische Kriterien zur Identifizierung eines psychogenen Thoraxschmerzes gibt es allerdings nicht [8].

\section{kurzgefasst}

Nicht-kardiale Ursachen eines Thoraxschmerzes können aufgrund ihrer typischen Symptomatik oft bereits klinisch vom akuten Koronarsyndrom abgegrenzt werden.

\section{Diagnostik}

$\checkmark$

Aufgrund der hohen Prävalenz des akuten Thoraxschmerzes in seinen oben dargestellten Ausprägungen und der Notwendigkeit einer raschen Differenzierung zwischen potenziell vital bedrohlichen und nicht vital gefährdenden Ursachen ist ein einfacher Algorithmus zur raschen Risikostratifizierung erforderlich ( Abb.1). Hier hat sich die Einführung spezieller Brustschmerzambulanzen (CPU) bewährt. Die Deutsche Gesellschaft für Kardiologie hat in ihrem Konsensuspapier [16] wesentliche Grundvoraussetzungen festgelegt. Neben einer genauen Anamnese und klinischen Untersuchung muss beim Leitsymptom Thoraxschmerz regelhaft innerhalb von 10 Minuten nach der Aufnahme ein 12-Kanal-EKG angefertigt und unverzüglich ärztlich ausgewertet werden. Darüber hinaus sind als Mindestanforderung an Labordiagnostik ein Troponin I/T-Test und die D-Dimere initial zu bestimmen. Sowohl EKG als auch Labor sollen nach spätestens 3-6 Stunden kontrolliert werden, sofern die Schmerzursachen nicht eindeutig als nicht-kardial eingestuft werden.

Eine Röntgenaufnahme des Thorax (idealerweise im Stehen in posterior-anteriorem Strahlengang) gehört bei nicht eindeutig kardialem Thoraxschmerz zur Basis-Bildgebung: Hierdurch können ein Pneumothorax sowie infiltrative Lungenparenchymerkrankungen diagnostiziert werden. Eine kapilläre Blutgasanalyse kann diese Diagnosen erhärten.

Eine transthorakale Echokardiographie ist zum Ausschluss weiterer Differenzialdiagnosen und zum Nachweis segmentaler Kinetikstörungen des linken Ventrikels bzw. Rechtsherzbelastungszeichen in der Frühphase durchzuführen.

\section{kurzgefasst}

12-Kanal-EKG, Troponin-Bestimmung und Röntgen-ThoraxAufnahme sind zentrale diagnostische Instrumente in der initialen Differenzialdiagnostik.

\section{Therapie}

$\checkmark$

Bei einem akuten Koronarsyndrom (ACS) basiert die Behandlung auf 4 wesentlichen Prinzipien. 


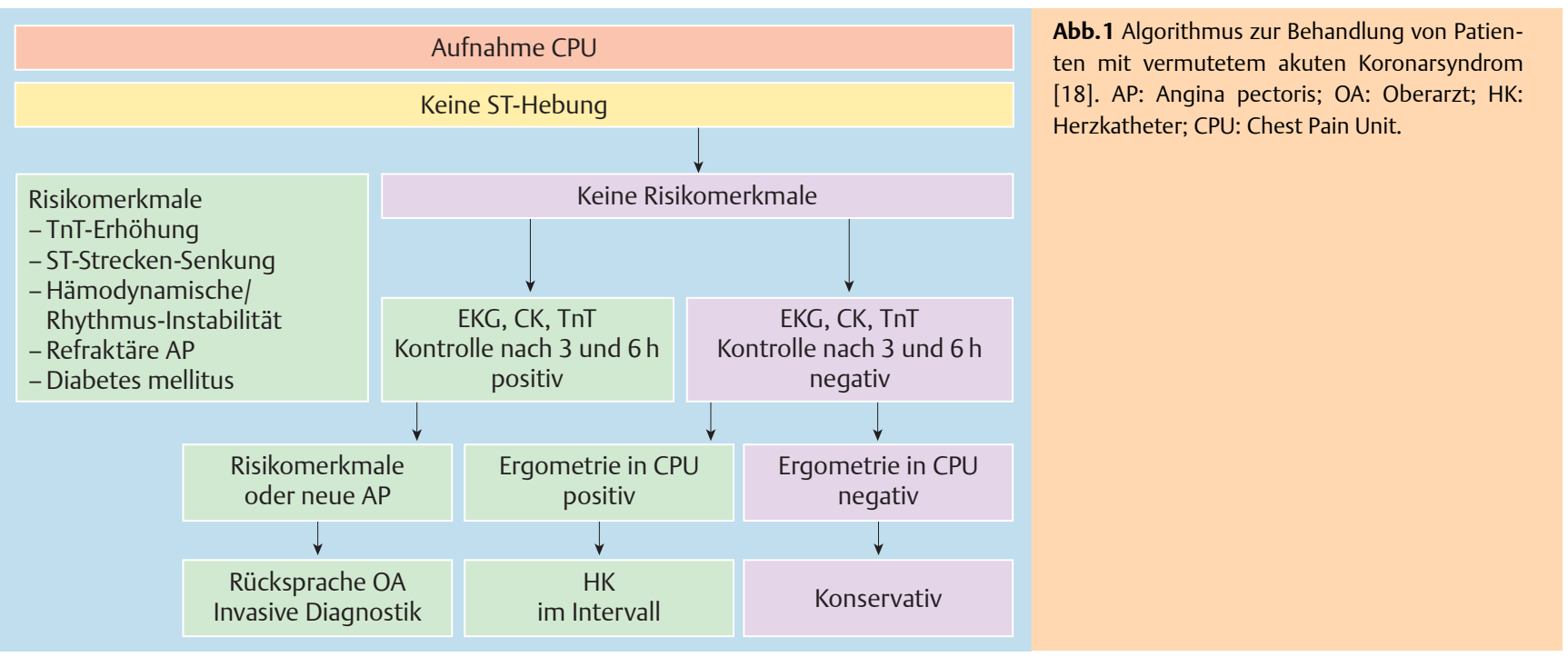

1.Antiischämische Therapie: Ziel ist die Senkung des myokardialen Sauerstoffverbrauches und die Optimierung der Perfusion (z.B. durch Beta1-selektive Betablocker, Nitrate oder Kalziumantagonisten).

2.Antikoagulation: Eine Antikoagulation ist zusätzlich zur obligaten Thrombozytenaggregationshemmung indiziert bei allen Patienten mit ACS (z.B. unfraktioniertes Heparin, Enoxaparin, Fondaparinux oder Bivalirudin).

3.Thrombozytenaggregationshemmung: Acetylsalicylsäure (ASS) senkt das Risiko für Tod und Myokardinfarkt bei instabiler Angina pectoris in einer Metaanalyse [1] um bis zu 8\% und gehört damit zur Basistherapie des ACS. Nach den aktuellen Leitlinien der ESC aus 2011 soll eine Einmal-Dosis von 150-300 mg unverzüglich nach Diagnosestellung gegeben werden, gefolgt von einer täglichen Erhaltungsdosis von $100 \mathrm{mg}$.

Das Thienopyridin Clopidogrel konnte in Kombination mit ASS $100 \mathrm{mg}$ in der CURE-Studie die Mortalität sowie nichttödliche Myokardinfarkte innerhalb der ersten 30 Tage um 20\% senken, wobei dieser Effekt bereits in den ersten 24 Stunden deutlich wurde. Das erhöhte Risiko schwerer Blutungen wird durch die Vorteile der Clopidogrel-Therapie aufgehoben. Beim ACS wird in den ESC-Leitlinien eine Loading-Dosis von $300 \mathrm{mg}$ empfohlen, gefolgt von einer täglichen Erhaltungsdosis von $75 \mathrm{mg}$. Höhere Loading-Dosen (600 bzw. $900 \mathrm{mg}$ ) führen zu einer schnelleren Plättchen-Inhibition (2-4 Stunden) und scheinen die Rate an Non-Respondern zu reduzieren [15]. Daher sollten Patienten, die eine primäre PCI erhalten, eine Loading-Dosis von $600 \mathrm{mg}$ erhalten [6].

Prasugrel ist ein Thienopyridin mit schnellerem Wirkeinstritt im Vergleich zu Clopidogrel (ca. 30 Minuten). Derzeit gibt es keine Hinweise auf Prasugrel-Non-Responder. In der TRITONTIMI 38-Studie wurde die Substanz mit einer Loading-Dosis von $60 \mathrm{mg}$, gefolgt von einer Erhaltungsdosis von $10 \mathrm{mg}$ täglich gegen Clopidogrel (300 mg Loading-Dosis, 75 mg Erhaltungsdosis) verglichen: Der kombinierte Endpunkt (Tod, nichttödlicher Myokardinfarkt, Schlaganfall) war unter Prasugrel signifikant geringer als unter Clopidogrel, v.a. infolge einer geringeren Rate an Myokardinfarkten (9,3 vs. 11,2\%). Die Blutungskomplikatio- nen waren im Gegensatz dazu unter Prasugrel signifikant höher ( 2,4 vs. $1,8 \%$ ). Die Substanz wird derzeit nicht empfohlen bei Patienten nach Schlaganfall, Alter $\geq 75$ Jahre und einem Körpergewicht unter $60 \mathrm{~kg}$ [27].

Mit Ticagrelor ist seit 2011 eine neue antithrombozytär wirksame Substanz auf dem Markt, die in der PLATO-Studie im Vergleich zur Standardtherapie mit Clopidogrel zusätzlich zur ASSTherapie eine weitere Reduktion von Mortalität und nichttödlichen Myokardinfarkten gezeigt hat, bei gleichem Blutungsrisiko [24]. Die aktuelle ESC-Leitlinie empfiehlt diese Therapie (Loading-Dosis $180 \mathrm{mg}$, Erhaltungsdosis $2 \times 90 \mathrm{mg}$ tgl.) für alle Patienten mit einem mittleren bis hohen Risiko für kardiovaskuläre Ereignisse (d.h. alle Troponin-positiven Patienten) unabhängig, ob eine invasive Koronar-Therapie oder eine medikamentös konservative Therapie verfolgt wird.

Eine Dosis-Anpassung bei Niereninsuffizienz ist bei keinem der genannten Thrombozyten-Aggregationshemmer erforderlich.

4.Revaskularisation: Neben einer symptomatischen Komponente (Beseitigung der Myokardischämie) steht unter prognostischen Aspekten die Revaskularisation zur Verhinderung eines persistierenden Myokardschadens mit fatalen Spätfolgen wie Herzinsuffizienz und ventrikulären Tachyarrhythmien im Vordergrund.

- Eine unverzügliche invasive Diagnostik (Koronarangiographie) ist bei Vorliegen eines ST-Hebungsinfarktes Therapie der Wahl mit einzeitiger perkutaner Koronarintervention (PCI) der Infarktarterie. Eine systemische Fibrinolyse ist nur indiziert bei Patienten mit Symptombeginn $<3$ Stunden, bei denen eine Koronarintervention nicht innerhalb von $90 \mathrm{Mi}-$ nuten nach Diagnosestellung durchgeführt werden kann [26]. Der Zeitpunkt der invasiven Diagnostik bei Patienten mit NSTEMI wird - adjustiert nach dem GRACE-Risiko-Score - festgelegt:

- Eine dringende invasive Diagnostik ( $<2$ Stunden nach Diagnosestellung) ist angezeigt bei Hochrisiko-Patienten mit therapierefraktärer oder rezidivierender Angina pectoris mit dynamischen ST-Streckenveränderungen, vital bedrohlicher Herzinsuffizienz, hämodynamischer Instabilität und ventrikulären Arrhythmien. 


\begin{tabular}{|c|c|c|c|c|c|}
\hline \multirow{2}{*}{$\begin{array}{c}\text { Mortalitätsrisiko } \\
\text { hoch } \\
(>15 \%)\end{array}$} & klin. Befund & RV-Dysfunktion & myok. Schaden & $\begin{array}{c}\text { Abb.2 Risikostratifizerung der akuten Lungenem- } \\
\text { bolie anhand der embolieabhängigen Frühmorta- } \\
\text { lität [1] }\end{array}$ \\
\hline $\begin{array}{c}\text { mittel } \\
(3-15 \%)\end{array}$ & + & + & + & $\begin{array}{c}\text { Thrombolyse } \\
\text { Embolektomie }\end{array}$ & \\
\hline $\begin{array}{c}\text { niedrig } \\
(<15 \%)\end{array}$ & - & + & + & $\begin{array}{c}\text { Rtationäre } \\
\text { Behandlung }\end{array}$ \\
\hline
\end{tabular}

- Eine früh-invasive Diagnostik (<24 Stunden nach Diagnosestellung) wird angestrebt bei Risikopatienten, die unter medikamentöser Therapie anhaltend beschwerdefrei sind, jedoch eine invasive Diagnostik erhalten sollen (TMF $\geq 4$ oder GRACE $>140$ Punkte).

- Eine elektiv-invasive Diagnostik (< 72 Stunden nach Diagnosestellung) ist indiziert bei Niedrig-Risiko-Patienten, die unter initialer medikamentöser Therapie anhaltend beschwerdefrei sind [6].

Kleine Pneumothoraces (Spaltbreite $<3 \mathrm{~cm}$ Pleurakuppen-Lungenparenchymabstand) bei klinisch stabilen Patienten sollten für 3-6 Stunden nach Diagnosestellung stationär beobachtet werden; kann in einer anschließenden Verlaufskontrolle im Röntgenbild kein Progress der Pneumothoraxgröße festgestellt werden, ist eine weitere ambulante Betreuung bei kurzfristiger radiologischer Verlaufskontrolle möglich. Bei einem großen Pneumothorax ( $\geq 3 \mathrm{~cm}$ ) ist eine stationäre Behandlung mit Anlage einer Thoraxsaugdrainage und aktivem Sog (in der Regel über ein Wasserschloss) indiziert. Eine radiologische Verlaufskontrolle ist frühestens nach 5-12 Stunden indiziert.

Bei Verdacht auf eine Lungenarterienembolie ist ein frühe Risikostratifizierung ebenfalls prognostisch bedeutsam: Routinemäßig werden in den meisten Kliniken D-Dimere bestimmt, Abbauprodukte des vernetzten Fibrins. Die Aussagekraft dieses Tests für die Diagnosestellung einer Lungenarterienembolie bzw. venösen Thromboembolie ist jedoch gering: Selbst mit modernen Testmethoden (ELISA-Test) liegt die Sensitivität nur bei ca. 40\%: Perrier untersuchte 280 Patienten und konnte nur bei $9 \%$ der Patienten mit pathologisch erhöhten D-Dimeren eine Thromboembolie nachweisen [17].

Hier haben sich Scoring-Systeme etabliert, die anhand des Vorliegens prädisponierender und klinischer Faktoren die Wahrscheinlichkeit einer Lungenarterienembolie erhärten sollen; im klinischen Alltag hat sich neben dem Genfer-Score vor allem der sogenannte Wells-Score etabliert ( Tab.2): Bei einem Punktwert $\leq 4$ liegt die klinische Wahrscheinlichkeit einer Lungenarterienembolie bei 2,8\%, wenn die D-Dimere negativ sind [25]. Neben klinischen Aspekten wie Hypotension oder Schock und biochemischen Parametern (z.B. Troponin) spielt in der Frühphase vor allem eine rasche kardiale Bildgebung zur Beurteilung der Rechtsherzfunktion eine wichtige Rolle: In den aktuellen Leitlinien der europäischen Gesellschaft für Kardiologie [22] wird ein einfacher Algorithmus dargestellt, der anhand der 3 oben erwähnten Kriterien das Mortalitätsrisiko klassifiziert ( Abb.2). Bei einem hohen Risiko liegt der Schwerpunkt der Akutbehandlung auf einer raschen Therapie, d.h. systemischen
Tab.2 Wells-Score zur Einschätzung der Wahrscheinlichkeit einer akuten Lungenembolie [25].

\begin{tabular}{|c|c|}
\hline Variable & Punkte \\
\hline \multicolumn{2}{|l|}{ Prädisponierende Faktoren } \\
\hline - stattgehabte tiefen Venenthrombose bzw. Lungenembolie & 1,5 \\
\hline $\begin{array}{l}\text { kürzlich zurückliegende Immobilisation bzw. chirurg. Eingriff } \\
\text { innerhalb der letzten } 4 \text { Wochen }\end{array}$ & 1,5 \\
\hline - Tumor-Erkrankung & 1 \\
\hline \multicolumn{2}{|l|}{ Symptome } \\
\hline - Hämoptysen & 1 \\
\hline \multicolumn{2}{|l|}{ Klinische Zeichen } \\
\hline - Herzfrequenz > 100/min & 1,5 \\
\hline - Zeichen einer tiefen Venenthrombose & 3 \\
\hline \multicolumn{2}{|l|}{ Klinische Entscheidung } \\
\hline - Keine alternative Diagnose außer Lungenembolie & 3 \\
\hline \multicolumn{2}{|l|}{ Wahrscheinlichkeit (3-Stufen) } \\
\hline - niedrig & $0-1$ \\
\hline - moderat & $2-6$ \\
\hline - hoch & $\geq 7$ \\
\hline \multicolumn{2}{|l|}{ Wahrscheinlichkeit (2-Stufen) } \\
\hline - Lungenembolie unwahrscheinlich & $0-4$ \\
\hline - Lungenembolie wahrscheinlich & $\geq 4$ \\
\hline
\end{tabular}

Thrombolyse (Empfehlungsgrad I B), wohingegen bei Patienten mit mittlerem und niedrigem Risiko der Schwerpunkt auf eine rasche Diagnose-Sicherung gelegt werden soll (Empfehlungsgrad Ila B). Als bildgebende Nachweis- bzw. Ausschluss-Verfahren kommt neben einer Pulmonalisangiographie (z.B. mittels Thorax-CT) die Ventilations-Perfusions-Szintigraphie (sog. V/QScan) infrage.

\section{kurzgefasst}

Bei akuten Koronarsyndromen ist neben einer raschen antithrombozytären Therapie die Koronarangiographie - im Bedarfsfall mit einzeitiger Koronarintervention - Therapie der Wahl. Einfache Risikoscores sind zur Prognose-Abschätzung und Festlegung von Therapie-Strategien hilfreich.

Autorenerklärung: Die Autoren erklären, dass sie keine finanziellen Verbindungen mit einer Firma haben, deren Produkt in dem Artikel eine wichtige Rolle spielt (oder mit einer Firma, die ein Konkurrenzprodukt vertreibt). 


\section{Konsequenz für Klinik und Praxis}

Die rasche diagnostische Abklärung bei akuten Thoraxschmerzen ist aufgrund der hohen Prävalenz potenziell lebensbedrohlicher Erkrankungen entscheidend. Chest Pain Units sind hier aufgrund einheitlicher Algorithmen sehr effizient.

Akute Koronarsyndrome liegen in ca. 25\% der Fälle vor und sollten zügig einer invasiven Diagnostik zugeführt werden. Evaluierte Risikoscores erleichtern in der initialen Diagnostik die Festlegung diagnostischer und therapeutischer Algorithmen.

Literatur

1 Antithrombotic Trialists' (ATT) Collaboration. Aspirin in the primary and secondary prevention of vascular disease: collaborative meta-analysis of individual participants from randomized trials. Lancet 2009; 373: 1849-1860

2 Banks PA et al. Practice guidelines in acute pancreatitis. Am J Gastroenterol 2006; 101: 2379-2400

3 Baumann MH, Strange C, Heffner JE et al. Management of Spontaneous Pneumothorax: An American College of Chest Physicians Delphi Consensus Statement. Chest 2001; 119: 590-602

4 Bösner $S$ et al. AWMF-Leitlinie „Brustschmerz“ der DEGAM. DEGAMLeitlinie Nr. 15. 2011; AMWF-Register Nr. 053/023

5 Fruergaard DP, Launbjerg J, Hesse B et al. The diagnosis of patients admitted with acute chest pain but without myocardial infarction. Eur Heart J 1996; 17: 1028-34

$6 \mathrm{Hamm}$ CW et al. ESC Guidelines for the management of acute coronary syndromes in patients presenting without persistent ST-segment elevation. Eur Heart J 2011; 32: 2999-3054

7 Höffken G, Lorenz J, Kern W et al. Epidemiologie, antimikrobielle Therapie und Management von erwachsenen Patienten mit ambulant erworbenen unteren Atemwegsinfektionen sowie ambulant erworbener Pneumonie. Pneumologie 2009; 63: e61-68

8 Huffmann JC, Pollack MH. Predicting panic disorder among patients with chest pain: an analysis of the literature. Psychosomatics 2003; 44: 222-236

9 Karnath B, Holden MD, Hussain N. Chest pain: Differentiating cardiac from noncardiac causes. Hosp Physician 2004; 40: 24-38

10 Kodolitsch Y. Clinical prediction of acute aortic dissection. Arch Int Med 2000; 160: 2977-2982

11 Konstantinides S, Janssens U, Mayer E et al. Kommentar zu den ESC-Leitlinien „Guidelines on diagnosis and management of acute pulmonary embolism“. Kardiologe 2009; 3: 272-282

12 Koop H, Schepp W, Müller-Lissner S et al. Gastroösophageale Refluxkrankheit - Ergebnisse einer evidenzbasierten Konsensuskonferenz der Deutschen Gesellschaft für Verdauungs- und Stoffwechselkrankheiten. Z Gastroenterologie 2005; 43: 163-194

13 Korn O, Onate JC, Lopez R. Anatomy of the Boerhaave syndrome. Surgery 2007; 141: 222-228

14 Miniati M, Prediletto R, Formichi B et al. Accuracy of clinical assesment in the diagnosis of pulmonary embolism. Am J Respir Crit Care Med 1999; 159: 864-871

15 Montalescot G, Sideris G, Meulemann C et al. A randomised comparison of high dose clopidogrel loading doses in patients with non-ST-segment elevation acute coronary syndromes. J Am Coll Cardiol 2006; 48: 931938

16 Perings S, Smetak N, Block $M$ et al. Konsensuspapier der Task Force „Brustschmerz-Ambulanz“ der Deutschen Gesellschaft für Kardiologie Herz- und Kreislaufforschung. Kardiologe 2010; 4: 208-213

17 Perrier A, Roy PM, Aujesky D et al. Diagnosing pulmonary embolism in outpatients with clinical assessment, D-Dimer measurement, venous ultrasound and helical computes tomography: a multicenter management study. Am J Med 2004; 116: 291-299

18 Post F, Genth-Zotz S, Münzel T. Aktueller Stellenwert einer Chest Pain Unit in Deutschland. Herz 2007; 5: 435-437

19 de Schipper JP, Pull ter Gunne AF, Oostvogel HJM et al. Spontaneous rupture of the oesophagus: Boerhaave's syndrome in 2008. Dig Surg 2009 26: $1-6$

20 Slater EE, DeSanctis $R W$. The clinical recognition of dissecting aortic aneurysm. Am J Med 1976; 60: 625-631

21 Stein PD, Henry JW. Clinical characteristics of patients with acute pulmonary embolism stratified according to their presenting syndromes. Chest 1997; 112: 974-979
22 Torbick A, Perrier A, Konstantinides $S$ et al. Guidelines on the diagnosis and management of acute pulmonary embolism. Eur Heart J 2008; 29: 2276-2315

23 Thygesen K et al. Universal definition of myocardial infarction. Eur Heart J 2007; 28: 2525-2538

24 Wallentin L, Becker RC, Budaj A et al. Ticagrelor versus clopidogrel in patients with acute coronary syndromes. N Engl J Med 2009; 361: 10451057

25 Wells PS, Anderson DR, Rodger M et al. Derivation of a simple clinical model to categorize patients probability of pulmonary embolism. Thromb Haemost 2000; 83: 416-420

26 Werdan $K, R u ß M$, Buerke $M$ et al. Deutsch-österreichische S3-Leitlinie „Infarktbedingter kardiogener Schock - Diagnose, Monitoring und Therapie“. Kardiologe 2011; 5: 166-224

27 Wiviott SD, Braunwald E, McCabe CH et al. Prasugrel versus clopidogrel in patients with acute coronary syndromes. N Engl J Med 2007; 357; 2001 2015 